population of nearly 67 million, Thailand has about 93000 new cases each year and an overall estimated TB prevalence of nearly 130000 cases. There are many health reports that healthcare workers have many infection exposures not only from Tuberculosis but also have other respiratory infections too.

Methods A cross-sectional survey was conducted during 20132016 among the healthcare workers. Data was collected through pre-employment examination from 1637 staffs (240 male and 1397 female) inclusive of clinical and non-clinical departments.

Results There were 35 cases $(2.13 \%)$ of healthcare workers reported abnormal result in chest X-ray (figure 1). The comparison between the lung abnormalities (1.4\%) and other abnormalities $(0.73 \%)$ was done. There were $65.8 \%$ had lung abnormalities, $28.5 \%$ had bone abnormalities and $5.7 \%$ had mild cardiomegaly. There were 8 cases $(34.7 \%)$ of lung abnormalities had diagnosed with lung tuberculosis. There were 3 active tuberculosis cases $(8.5 \%)$ had to receive treatment prior to employment, and none of them had Multidrug-resistant TB (MDR TB). There were 4 cases (11.4\%) diagnosed with old tuberculosis and only one case (2.8\%) was NTM (Nontuberculous mycobacteria).There were $43.4 \%$ of lung abnormalities had small pulmonary nodule, $8.7 \%$ had fibrosis, $4.3 \%$ had cystic-like lesion. There were 8 cases $(80 \%)$ of bone abnormalities had diagnosed with mild scoliosis and did not typically expect to cause problems.

Conclusions The pre-employment examination of healthcare workers is as important as any other occupations. The chest $\mathrm{x}$-ray screening is cheap and relatively safe method. It might be helpful not only for their health but also useful to prevent patients from many communicable diseases.

\section{EVALUATION OF THE EFFICACY OF RISK MANAGEMENT STRATEGIES IN THE PROTECTION OF HEALTHCARE WORKERS HANDLING CYTOTOXIC DRUGS}

${ }^{1} \mathrm{~S}$ Kelly* ${ }^{2}$ A Reid. ' Specialist Registrar in Occupational Medicine, Tallaght Hospital, Dublin, Ireland; ' Consultant Occupational Physician, Tallaght Hospital and Coombe Women and Infants University Hospital, Dublin, Ireland

\subsection{6/oemed-2018-ICOHabstracts.961}

Introduction Cytotoxic drugs are used as part of cancer chemotherapy regimens in the healthcare setting and are recognised occupational hazards. Acceptable levels of exposure have not been established therefore national guidelines advocate levels as low as reasonably possible. This aim of this study was to establish whether training and reminders regarding work practices would limit occupational exposure to cytotoxic drugs. Platinum was chosen as the index cytotoxic drug due to its frequent use and validated analysis methods. Initial studies performed in 2007 and 2012 in our instituion found that focused training on the appropriate handling of cytotoxic agents brought about a reduction in occupational exposure to the drug.

Methods A prospective study was carried out in the oncology day ward and pharmacy aseptic compounding unit of a tertiary level hospital. Urinalysis for urinary platinum was carried out on 10 staff members, including one control. End of shift, end of week samples were collected from a randomly selected group of staff. Samples were sent to an internationally accredited laboratory for analysis. A review of work processes was also performed.

Result The mean urinary platinum level was $26.19 \mathrm{nmol} / \mathrm{mol}$ creatinine (range $15.89-91.42 \mathrm{nmol} / \mathrm{mol}$ creatinine), which is well above the reference level for the unexposed worker of $10 \mathrm{nmol} / \mathrm{mol}$. The control was also high at $49.71 \mathrm{nmol} / \mathrm{mol}$. Mean platinum concentration values increased by a factor of 11 from the 2012 study.

Discussion These high urinary platinum results were unexpected. There had been no changes in equipment or work practices since the last study performed in 2012. As the control result was also high this was most likely a laboratory error or contamination rather than true high occupational exposures. To further evaluate this, we repeated testing and these results are pending (expected end of July).

\section{HOSPITAL ADMISSIONS RELATED TO INJURY AMONG SOUTH KOREA FEMALE FIREFIGHTERS: KOREAN NATIONAL FIREFIGHTER COHORT STUDY}

${ }^{1} \mathrm{Ji}$ Young Song, ${ }^{2}$ Gab-Sik Shin, ${ }^{2}$ Min-gi Kim*, ${ }^{3}$ Yeon-Soon Ahn. ${ }^{1}$ Department of Nursing, Korea University, Seoul, Republic of Korea; ${ }^{2}$ Departments of Occupational and Environmental Medicine, Dongguk University Gyeongju Hospital, Gyeongju, Republic of Korea; ${ }^{3}$ Department of Occupational and Environmental Medicine, Dongguk University Ilsan Hospital, 29 Dongguk-no, Ilsandong-gu, Goyang 410-773, Republic of Korea

\subsection{6/oemed-2018-ICOHabstracts.962}

Introduction The main objective of this study was to ascertain whether hospital admission related to the injury in all South Korea female firefighters has increased compared to general population and to find out if there is any difference according to the job types and work duration.

Method The present study compared hospital admissions for injury, based on ICD-10 codes in Korean female firefighters with those in the general Korean population. Standardised admission ratios (SARs) and their 95\% confidence intervals (CI) were calculated by using person-years and mortality computation software.

Result Compared to the general female population, all of injury $(\mathrm{SAR}=1.57,95 \% \mathrm{CI}: 1.24$ to 1.96$)$ as well as for injury to abdomen, lower back, lumbar spine and pelvis $(\mathrm{SAR}=2.78$, 95\% CI: 1.81 to 4.07 ) in female firefighters were significantly high. The SARs of female firefighter whose working duration $<10$ years in fire suppression $(\mathrm{SAR}=3.34,95 \% \mathrm{CI}$ : 1.52 to 6.35), Emergency Medical Service (EMS) (SAR=2.86, 95\% CI: 1.35 to 5.27 ) and office ( $\mathrm{SAR}=3.69,95 \% \mathrm{CI}: 1.68$ to 7.00 ) were significantly high. In addition, the SAR values for injuries to knee and lower leg $(\mathrm{SAR}=2.48,95 \% \mathrm{CI}: 1.181$ to $4.55)$ in female Firefighters working in the EMS were significantly high

Discussion Efforts should be made to prevent lumbar injury in female firefighters with short work duration. Female firefighter who working in EMS need to carful about lower body injuries Further study is need to identify and prevent injury the occurrence of injury in female firefighters.

Abbreviations SAR, standardised admission ratio; CI, confidence interval; EMS, Emergency Medical Service

Acknowledgments This research was supported by a grant [2017-MPSS25-031-01010000-2017] through the National Disaster Management Research Institute funded by Ministry of Public Safety and Security of Korean government. 